a comparison can be dangerous in both directions. If a comparison of fatality rates is applied to countries with a strong containment of the COVID-19 pandemic, the severity of COVID-19 may be underrated, which can be potentially disastrous, especially in the presence of severe comorbidities. On the other hand, presenting severe acute respiratory coronavirus virus 2 (SARS-CoV-2) as a virus with much higher fatality rates can underrate the severity of the seasonal flu, with negative effects, for example, on flu vaccination rates.

According to Faust et al, ${ }^{1}$ the root of the confusion is a knowledge gap regarding how influenza and COVID-19 data are reported. Covid-19 data are actual numbers, whereas influenza data are only calculated estimates. This important point cannot be overstated. Faust et al compared COVID-19 death counts to influenza death counts over past seasons. They reported that a 1-week COVID-19 death rate in April 2020 was 9.5- to 44.1-fold greater than the peak week of influenza deaths during any of the past 7 influenza seasons. They also analyzed the case of Diamond Princess and reported that even the adjusted CFR of $0.5 \%$ "would still be 5 times the commonly cited CFR of adult seasonal influenza." Moreover, Faust et al concluded that to understand the true threat to public health from COVID-19, comparisons with seasonal influenza should be made using an apples-to-apples comparison. ${ }^{1}$

Although their facts are accurate, such comparisons may underrate the severity of seasonal influenza; rather, they are apples-topears comparisons. First and most importantly, COVID-19 is an ongoing pandemic, whereas influenza is largerly caused by endemic strains of several influenza virus subtypes that have circulated over decades as seasonal flu. These viral strains cause more or less severe epidemics annually. This evolving COVID-19 pandemic, caused by a new, previously unknown virus that has overwhelmed healthcare systems and caused shortage of medical supplies in almost every country of the world, cannot be directly compared to the seasonal outbreaks of an endemic disease caused by a well-studied virus for which vaccine protection is available for the main strains.

It would be more reasonable to compare 2 pandemics with each other. Several studies of this type exist; most compare the current pandemic with the 1918-19 influenza (Spanish flu) pandemic. He et al ${ }^{2}$ adopted the conventionally accepted CFR of $2 \%$ for 1918-19 influenza and reported comparable fatality rates in the United Kingdom. ${ }^{2}$ However, whether 2 pandemics that occurred 100 years apart can be compared on an apples-to-apples basis is also questionable. For example, suspected cases of the Spanish flu were not confirmed by laboratory tests and therefore the infection fatality rate (IFR; ie, proportion of deaths among all infected individuals of the Spanish flu) has been compared with the CFR (ie, proportion of death among individuals with laboratory-confirmed disease). Furthermore, fatality rates do not reflect the proportion of the world population infected. The Spanish flu infected $\sim 33 \%$ of the world population at the time.

Another candidate for a direct comparison could be the last global influenza pandemic, which occurred in 2009, due to the swine-origin influenza A virus subtype H1N1. According to the World Health Organization, the total number of laboratoryconfirmed pandemic A(H1N1) cases was $491,382,{ }^{3}$ including 18,449 deaths. ${ }^{4}$ This represents an overall CFR of $3.75 \%$, with a mean age at death of 37.4 years. ${ }^{5}$ The WHO emphasized "that the reported number of fatal cases is an under representation of the actual numbers as many deaths are never tested or recognized as influenzarelated." Although the CFR of the "fairly mild 2009 influenza pandemic" 5 is apparently much higher than that of the current COVID19 pandemic, such a comparison is, in fact, also debatable. For example, the WHO declared the counting of individual cases as no longer essential only a few months after declaring the $\mathrm{H} 1 \mathrm{~N} 1$ pandemic.

In summary, there is no need to directly compare COVID-19 and influenza in terms of fatality rates to prove the severity of the current pandemic. Both are harmful, dangerous, and potentially disastrous diseases, and they should be treated with the utmost respect.

Acknowledgments.

Financial support. No financial support was provided relevant to this article.

Conflicts of interest. All authors report no conflicts of interest relevant to this article.

\section{References}

1. Faust JS, Del Rio C. Assessment of deaths from covid-19 and from seasonal influenza. JAMA Intern Med 2020;180:1045-1046.

2. He D, Zhao S, Li Y, Cao P, Gao D, Lou Y, Yang L. Comparing COVID-19 and the 1918-19 influenza pandemics in the United Kingdom. Int J Infect Dis 2020;98:67-70.

3. Weekly virological update on 05 August 2010. World Health Organization website. https://www.who.int/csr/disease/swineflu/laboratory06_08_2010/ en/. Published 2010. Accessed December 23, 2020.

4. Pandemic (H1N1) 2009 - update 112. World Health Organization website. https://www.who.int/csr/don/2010_08_06/en/. Published 2010. Accessed Dec 23, 2020.

5. Petersen E, Koopmans M, Go U, et al. Comparing SARS-CoV-2 with SARS-CoV and influenza pandemics. Lancet Infect Dis 2020;20:e238-e244.

\title{
Uptake of treatment practice standards during a pandemic in an academic medical system
}

\author{
Maria Tate $\mathrm{MS}^{1}$, Jeannie D. Chan PharmD, MPH ${ }^{1,2}$, Christine Johnston MD, MPH${ }^{2}$, Shireesha Dhanireddy $\mathrm{MD}^{2}$, Margaret \\ L. Green MD, MPH² and Rupali Jain PharmD ${ }^{1,2}$ (D) \\ ${ }^{1}$ University of Washington School of Pharmacy, Seattle Washington and ${ }^{2}$ Division of Allergy and Infectious Disease, University of Washington School of Medicine, \\ Seattle, Washington
}

\author{
Author for correspondence: Rupali Jain, E-mail: rupali@uw.edu \\ Cite this article: Tate M, et al. (2022). Uptake of treatment practice standards during a \\ pandemic in an academic medical system. Infection Control \& Hospital Epidemiology, 43: \\ 959-961, https://doi.org/10.1017/ice.2021.137
}

To the Editor - The first case of community spread of severe acute respiratory coronavirus virus 2 (SARS-CoV-2) in the United States was reported in Washington state in late January $2020 .{ }^{1}$ During the early stage of the pandemic, therapeutics were emerging at a rapid 
Incorporation of COVID-19 Therapeutics at UW Medicine

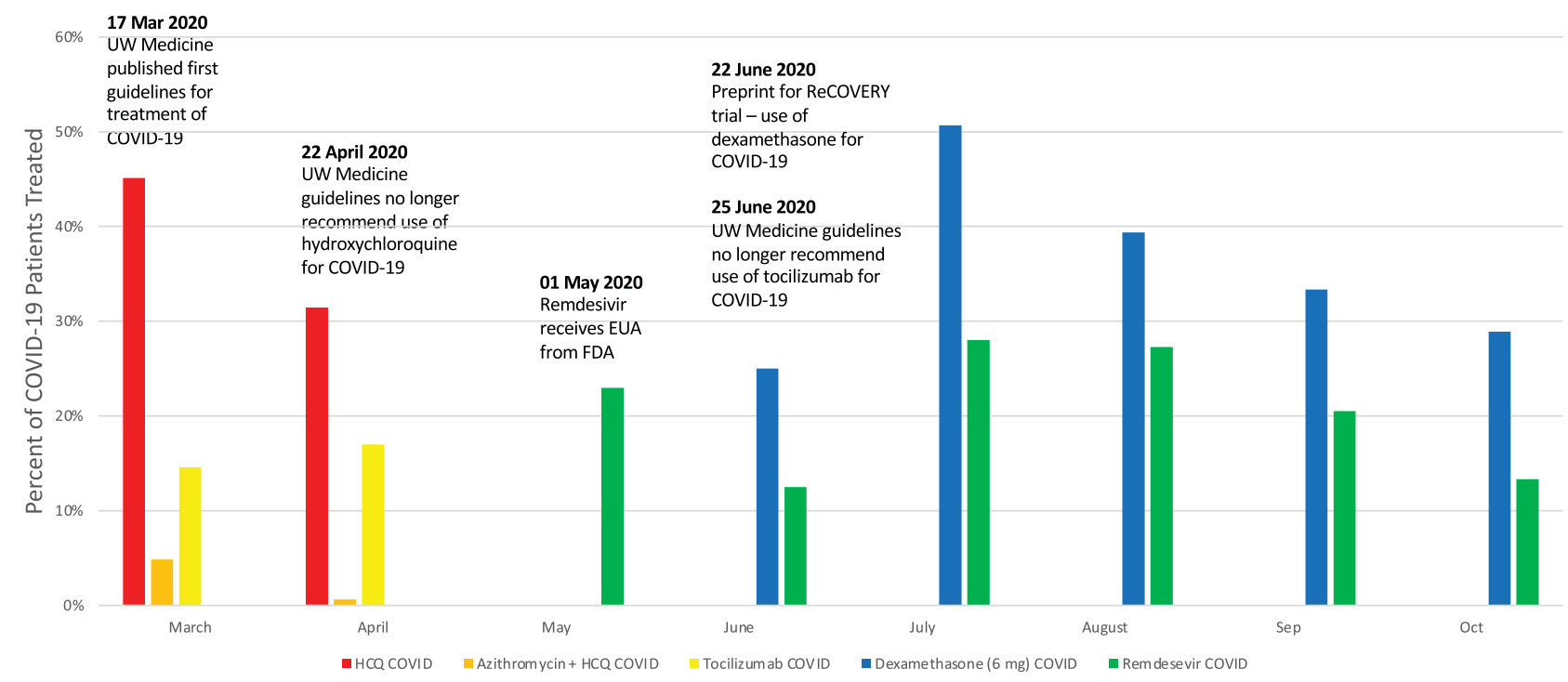

\begin{tabular}{|l|l|l|l|l|l|l|l|l|}
\hline $\begin{array}{l}\text { Number of COVID } \\
\text { patients }\end{array}$ & March: 144 & April: 159 & May: 61 & June: 32 & July: 75 & August: 66 & September: 39 & October: 45 \\
\hline
\end{tabular}

Fig. 1. Timeline of implementation of COVID-19 therapeutics and prescribing patterns at UW Medicine.

pace, often without peer review, and evidenced-based national guidelines were not yet available. To develop and disseminate guidance for safe and consistent care using data available at the time, the University of Washington (UW) Medicine clinicians convened a practice guidelines committee, comprising faculty from infectious diseases, pulmonary/critical care, cardiology, hematology, internal medicine and pharmacy. ${ }^{2}$ We utilized World Health Organization Guidance for Managing Ethical Issues in Infectious Disease Outbreaks, which provides a framework for ethical use of unproven interventions during pandemics, to guide our recommendations in the absence of rigorous evidence from clinical trials. ${ }^{3}$ The first internal guideline was published on March 17, 2020, and updates were made every 1-2 weeks thereafter. After publication of the Infectious Diseases Society of America (IDSA) guidelines on April 11, 2020, and the National Institutes of Health (NIH) guidelines on May 12, 2020, ${ }^{4,5}$ we coordinated with national guidelines, but we continued to provide guidance on implementation of therapeutics and integrated care pathways into the electronic medical record to facilitate the ordering and monitoring of these therapies. We also incorporated ongoing clinical trials as they became available within our system in the internal guidelines as a resource for clinicians to help identify potential candidates for study enrollment. We sought to review the impact of our local guidelines on prescribing patterns for COVID-19 therapeutics at our health system.

The UW Medicine system comprises 3 hospitals and > 300 clinics across the Puget Sound region. As part of a quality improvement initiative, pharmacy records from the UW Medicine hospitals (Harborview, University of Washington Medical Center, Montlake and Northwest campuses) were queried regarding the use of hydroxychloroquine (HCQ), tocilizumab, lopinavirritonavir, interferon, ivermectin, dexamethasone (6 $\mathrm{mg})$, and remdesivir from March 1 to October 15, 2020. Records of patients who received HCQ were also reviewed for azithromycin use. The clinical indication was obtained from provider notes and categorized into COVID-19 versus non-COVID-19 indications.
Surveillance testing for SARS-CoV-2 was performed for all patients upon admission starting April 13, 2020. The number of asymptomatic and symptomatic patients with COVID-19 were obtained from hospital census data for review.

We reviewed 1,006 pharmacy records of patients receiving the interventions. Overall, 333 patients were prescribed HCQ, 156 were prescribed tocilizumab, 110 were prescribed dexamethasone (6 mg), 71 were prescribed remdesivir, 73 were prescribed ivermectin, and 4 were prescribed interferon for various indications. We examined the timeline of the recommendations in local guidelines with drug utilization (Fig. 1). In March and April, 71\% of HCQ use and $62 \%$ of tocilizumab use were attributed to COVID-19, respectively. Azithromycin plus HCQ was prescribed for 8 patients. Consistent with the guidelines, no patients were prescribed ivermectin, interferon, or lopinavir-ritonavir for COVID-19. When internal guidelines recommended against HCQ and tocilizumab except in the context of clinical trials on April 22 and June 25, respectively, no patients were prescribed these agents for a COVID-19 indication thereafter.

After the US Food and Drug Administration (FDA) issued emergency use authorization for remdesivir based on preliminary data from the Adaptive COVID-19 treatment trial (ACTT-1) on May 1, 2020, this antiviral was incorporated into practice. ${ }^{6,7}$ Due to the scarcity of remdesivir early in the pandemic, a clinical allocation team consisting of pulmonary/critical care, infectious diseases, pharmacy, ethics, and medical leadership drafted clinical criteria for remdesivir to ensure appropriate use and equitable distribution. Providers submitted a request for remdesivir with deidentified data via Research Electronic Data Capture (REDCap), ${ }^{8}$ and a clinical decision was made by the allocation team within 24 hours of the request. Among the 318 patients admitted with COVID-19 between May 1 and October 15, 2020, 71 patients (22\%) received remdesivir with the approval of the clinical allocation team. The median duration was 5 days for remdesivir (range, 1-10 days). Dexamethasone was first recommended on June 23, 2020, after the preprint release of the RECOVERY trial. ${ }^{9}$ Among 
the 225 patients admitted with COVID-19 between July and October 2020, 98 patients (44\%) received dexamethasone.

In the face of a pandemic with rapidly evolving data, internal guidelines had a significant impact on the prescribing patterns of the wide range of empiric COVID-19 therapeutics because the validity of these agents was still being evaluated and national consensus guidelines were not yet available. Successful implementation of local guidelines is attributed to a small number of dedicated COVID-19 providers, daily huddles with providers, teaching conferences, and incorporation of care-plan pathway into the electronic medical record. We acknowledge that the lower volume of COVID-19 cases in our region provided a more conducive environment for a meticulous and thoughtful process for guidelines development and implementation. These data exemplify the need for local champions to synthesize available evidence with scientific rigor and implement national guidelines. The limitations of this study include the lack of direct attribution of the internal guidelines to prescribing patterns, but the distribution of medication utilization over time suggests that providers integrated local guidance to inform treatment decisions.

Acknowledgments. We acknowledge the members of the UW Medicine Treatment Guidelines Committee and Clinical Allocation Teams: Fred Buckner, Jeannie Chan, Guang-Shing Cheng, Shireesha Dhanireddy, Terry Gernsheimer, Margaret Green, Robert Harrington, Josh Hill, Rupali Jain, Christine Johnston, H. Nina Kim, David Koelle, Mark Mayo, Manoj Menon, Sylvia LaCourse, Paul Pottinger, Anneliese Schleyer, Mark Tonelli, Alpana Waghmare, Anna Wald, Anne Woolfrey, and Mark Wurfel. We also thank Semhar Nega-Asrat, and Brennen Yasuda for completing the data collection.

Financial support. No financial support was provided relevant to this article.
Conflicts of interest. C.J. has received funding from Gilead for consultation on a non-COVID-19 related project. All other authors report no conflicts of interest relevant to this article.

\section{References}

1. Bedford T, Greninger AL, Roychoudhury P, et al. Cryptic transmission of SARS-CoV-2 in Washington state. medRxiv 2020. doi: 10.1101/2020.04.02. 20051417.

2. Murad MH. Clinical practice guidelines: a primer on development and dissemination. Mayo Clin Proc 2017;92:423-433.

3. World Health Organization. Guidance for managing ethical issues in infectious disease outbreaks. Geneva, World Health Organization, 2016. https://apps. who.int/iris/handle/10665/250580. Published 2016. Accessed March 25, 2021.

4. Bhimraj A, Morgan RL, Shumaker AH, et al. Infectious Diseases Society of America guidelines on the treatment and management of patients with COVID-19. Clin Infect Dis 2020; ciaa478.

5. COVID-19 Treatment Guidelines Panel. Coronavirus disease 2019 (COVID-19) treatment guidelines. National Institutes of Health website. https://www.covid19treatmentguidelines.nih.gov/. Accessed January 20, 2021.

6. Beigel JH, Tomashek KM, Dodd LE, et al. Remdesivir for the treatment of COVID-19: final report. N Engl J Med 2020;383:1813-1826.

7. Coronavirus (COVID-19) update: FDA issues emergency use authorization for potential COVID-19 treatment. US Food and Drug Administration website. https://www.fda.gov/news-events/press-announcements/coronaviruscovid-19-update-fda-issues-emergency-use-authorization-potential-covid-19treatment. Published May 1, 2020. Accessed December 22, 2020.

8. Harris PA, Taylor R, Minor BL, et al. The REDCap consortium: building an international community of software partners. J Biomed Inform 2019. doi: $10.1016 /$ j.jbi.2019.103208.

9. RECOVERY Collaborative Group, Horby P, Lim WS, et al. Dexamethasone in hospitalized patients with COVID-19-preliminary report. N Engl J Med 2021;384:693-704.

\title{
The first use of face mask in the history of medicine
}

\author{
Ali Taghizadieh MD¹, Javad Ghazi-Sha'rbaf $\mathrm{PhD}^{2}$, Reza Mohammadinasab $\mathrm{PhD}^{3}$ (1) and Saeid Safiri PhD ${ }^{4,5}$ (1) \\ ${ }^{1}$ Tuberculosis and Lung Disease Research Center, Tabriz University of Medical Sciences, Tabriz, Iran, ${ }^{2}$ Department of Islamic History and Civilization, Faculty of \\ Theology, Azarbaijan Shahid Madani University, Tabriz, Iran, ${ }^{3}$ Department of History of Medicine, School of Traditional Medicine, Tabriz University of Medical \\ Sciences, Tabriz, Iran, ${ }^{4}$ Social Determinants of Health Research Center, Department of Community Medicine, Faculty of Medicine, Tabriz University of Medical \\ Sciences, Tabriz, Iran and ${ }^{5}$ Rahat Breath and Sleep Research Center, Aging Research Institute, Tabriz University of Medical Sciences, Tabriz, Iran
}

To the Editor-Since human life began, epidemics have been a threat to human health. ${ }^{1}$ Today, coronavirus disease 2019 (COVID-19) has spread almost all over the entire world, and apparently one of the most important ways to prevent the transmission of severe acute respiratory coronavirus virus 2 (SARS-CoV-2) is by wearing a face mask. ${ }^{2}$ A face mask largely prevents the transfer of airborne particles to the human respiratory system and can therefore play a significant role in preventing the spread respiratory viruses during epidemics. ${ }^{3,4}$

Interestingly, the mask has been used since ancient times in Iran; however, it should not be confused with the clothing used for women's face veil. The word equivalent to the mask in ancient and medieval Iran was panam or pandam, which is also mentioned

Authors for correspondence: Reza Mohammadinasab and Saeid Safiri. Emails: Rmn. nasab@tbzmed.ac.ir; safiris@tbzmed.ac.ir

Cite this article: Taghizadieh A, et al. (2022). The first use of face mask in the history of medicine. Infection Control \& Hospital Epidemiology, 43: 961-962, https://doi.org/ $10.1017 /$ ice.2021.157 in the Avesta, ${ }^{5}$ the holy book of Zoroastrians, in the religious rituals of the followers of the ancient prophet of Iran. ${ }^{6}$ The Iranian word panam is used in most middle and modern Iranian languages and dialects. This word in the Avestan language means holder and keeper against something, and the Pahlavi Persian language includes the words padam, pandam, and phanom.

According to Pahlavi texts, the panam, now called the veil by Iranian Zoroastrians, refers to 2 pieces of white cotton cloth that are hung over the mouth and tied with 2 ribbons at the back. This small cotton curtain, which according to the Pahlavi interpretation of the description in the Avesta, should be 2 inches below the mouth and should be used when the priest performs a religious ceremony in front of the sacred fir. In fact, the use of panam was to prevent the breath and steam of the mouth, which was considered polluted, from reaching the sacred element of fire. Therefore, it was a religious device of priests. ${ }^{6}$

Also, ancient Iranians who were honored by the king had wear a panam in front of their mouths to show respect for the king. 